\title{
How to Characterize Individual Nano-Size Liposomes with Simple Self-Calibrating Fluorescence Microscopy
}

Mortensen, Kim I.; Tassone, Chiara; Ehrlich, Nicky; Andresen, Thomas L.; Flyvbjerg, Henrik

\section{Published in:}

Nano Letters

Link to article, DOI:

10.1021/acs.nanolett.7b05312

Publication date:

2018

Document Version

Peer reviewed version

Link back to DTU Orbit

Citation (APA):

Mortensen, K. I., Tassone, C., Ehrlich, N., Andresen, T. L., \& Flyvbjerg, H. (2018). How to Characterize Individual Nano-Size Liposomes with Simple Self-Calibrating Fluorescence Microscopy. Nano Letters, 18(5), 2844-2851. https://doi.org/10.1021/acs.nanolett.7b05312

\section{General rights}

Copyright and moral rights for the publications made accessible in the public portal are retained by the authors and/or other copyright owners and it is a condition of accessing publications that users recognise and abide by the legal requirements associated with these rights.

- Users may download and print one copy of any publication from the public portal for the purpose of private study or research.

- You may not further distribute the material or use it for any profit-making activity or commercial gain

- You may freely distribute the URL identifying the publication in the public portal 


\section{How to Characterize Individual Nano-Size Liposomes with Simple Self-Calibrating Fluorescence Microscopy}

Kim I. Mortensen, Chiara Tassone, Nicky Ehrlich, Thomas L. Andresen, and Henrik Flyvbjerg

Nano Lett., Just Accepted Manuscript • DOI: 10.1021/acs.nanolett.7b05312 • Publication Date (Web): 03 Apr 2018

Downloaded from http://pubs.acs.org on April 6, 2018

\section{Just Accepted}

"Just Accepted" manuscripts have been peer-reviewed and accepted for publication. They are posted online prior to technical editing, formatting for publication and author proofing. The American Chemical Society provides "Just Accepted" as a service to the research community to expedite the dissemination of scientific material as soon as possible after acceptance. "Just Accepted" manuscripts appear in full in PDF format accompanied by an HTML abstract. "Just Accepted" manuscripts have been fully peer reviewed, but should not be considered the official version of record. They are citable by the Digital Object Identifier (DOI@). "Just Accepted" is an optional service offered to authors. Therefore, the "Just Accepted" Web site may not include all articles that will be published in the journal. After a manuscript is technically edited and formatted, it will be removed from the "Just Accepted" Web site and published as an ASAP article. Note that technical editing may introduce minor changes to the manuscript text and/or graphics which could affect content, and all legal disclaimers and ethical guidelines that apply to the journal pertain. ACS cannot be held responsible for errors or consequences arising from the use of information contained in these "Just Accepted" manuscripts. 


\title{
How to Characterize Individual Nano-Size
} Liposomes with Simple Self-Calibrating Fluorescence Microscopy

\author{
Kim I. Mortensen, ${ }^{* \dagger}{ }^{\dagger}$ Chiara Tassone, ${ }^{\dagger}$ Nicky Ehrlich, ${ }^{\dagger, \dagger}$ Thomas L. Andresen, ${ }^{\dagger}$ and \\ Henrik Flyvbjerg ${ }^{\dagger}$ \\ $\dagger$ Department of Micro- and Nanotechnology, Technical University of Denmark, Kongens \\ Lyngby, DK-2800, Denmark \\ $\ddagger$ Current address: Department of Chemical and Biochemical Engineering, Technical \\ University of Denmark, Kongens Lyngby, DK-2800, Denmark \\ E-mail: kim.mortensen@nanotech.dtu.dk
}

Phone: +4545256309

\begin{abstract}
Nano-size lipid vesicles are used extensively at the interface between nanotechnology and biology, e.g. as containers for chemical reactions at minute concentrations and vehicles for targeted delivery of pharmaceuticals. Typically, vesicle samples are heterogeneous as regards vesicle size and structural properties. Consequently, vesicles must be characterized individually to ensure correct interpretation of experimental results. Here we do that using dual-color fluorescence labeling of vesicles - of their lipid bilayers and lumens, respectively. A vesicle then images as two spots, one in each color channel. A simple image analysis determines the total intensity and width of each spot. These four data all depend on the vesicle radius in a simple manner for vesicles that


are spherical, unilamellar, and optimal encapsulators of molecular cargo. This permits identification of such ideal vesicles. They in turn enable calibration of the dual-color fluorescence microscopy images they appear in. Since this calibration is not a separate experiment but an analysis of images of vesicles to be characterized, it eliminates the potential source of error that a separate calibration experiment would have been. Non-ideal vesicles in the same images were characterized by how their four data violate the calibrated relationship established for ideal vesicles. In this way, our method yields size, shape, lamellarity, and encapsulation efficiency of each imaged vesicle. Applying this procedure to extruded samples of vesicles, we found that, contrary to common assumptions, only a fraction of vesicles are ideal.

\section{Keywords}

liposomes; vesicles; single particle analysis; dual-color fluorescence microscopy; lamellarity; encapsulation efficiency

\section{Main Text}

Phospholipid vesicles have been studied extensively over the last four decades, e.g. as model systems for biomembranes for their physicochemical properties, ${ }^{1}$ as environments for reconstitution and study of transmembrane proteins, ${ }^{2,3}$ as vessels for chemical reactions in small volumes, ${ }^{4,5}$ for engineering of exosomes, ${ }^{6}$ and as vehicles for delivery of nutrients. ${ }^{7}$ Vesicles in these studies have diameters from $5 \mu \mathrm{m}$ down to $100 \mathrm{~nm}$.

The smaller vesicles, with diameters near $100 \mathrm{~nm}$, are also of considerable interest as

capsules containing pharmaceuticals, e.g. in targeted chemotherapy. ${ }^{6,8-12}$ This vesicle size ${ }^{8,13}$ facilitates efficient transport to the intended target, prevents leaking on the way, facilitates cellular internalization, but is near the diffraction limit of optical microscopy, which hampers inspection and characterization of vesicles. 
Typically, vesicles in a sample differ in size, shape, lipid composition, lamellarity, and encapsulation efficiency. ${ }^{13-20}$ Consequently, one cannot interpret experiments correctly by assuming that vesicles are uni-lamellar and/or monodisperse; one needs to know the properties of individual vesicles. To this end, available bulk-level methods of characterization are useless. Instead, electron microscopy, atomic force microscopy, flow cytometry, and fluorescence microscopy may all serve this purpose. ${ }^{13}$

Fluorescence microscopy offers a particularly flexible and convenient solution for singleparticle characterization: ${ }^{13}$ particles can be studied in native conditions, with high throughput, dynamically if desired, and good fluorescent labels are abundant. Several microscopy modalities are available, such as laser-scanning confocal microscopy, ${ }^{15}$ epi-fluorescence microscopy ${ }^{16}$ and total internal reflection fluorescence (TIRF) microscopy. ${ }^{21}$ Indeed, singleparticle fluorescence-based methods have reported on size distributions of populations of vesicles, ${ }^{15,16,22}$ lamellarity, ${ }^{14,17}$ and encapsulation efficiency. ${ }^{19,23}$

Typically, the size of an individual vesicle is quantified using the total intensity measured from fluorophores in its bilayers: If a vesicle is spherical and unilamellar, that intensity is proportional to the square of the radius of the vesicle, which allows calculation of the latter, in principle. ${ }^{14-16,22,23}$ Multiple issues with established approaches remain, however:

(i) The constant of proportionality is unknown, unfortunately, without separate calibration experiments. Thus, cryogenic transmission electron microscopy (cryo-TEM) and dynamic light scattering have both been used to independently determine the sizes of vesicles in samples. The constant of proportionality is chosen such that the mode of the distribution of vesicle sizes in the characterization is identical to the mode of the distribution of vesicle sizes in the calibration experiment. ${ }^{15,16,23}$ Using the same basic approach, the diffusion coefficient of individual vesicles measured by single particle tracking has been used to determine their sizes and, in turn, the unknown constant. ${ }^{22}$ Alternatively, the widths of imaged spots have been related to vesicle sizes. This approach requires deconvolution with the microscope's point spread function (PSF), however, with the PSF determined in a separate calibration 
experiment using small, fluorescent nanoparticles. ${ }^{14}$ In all cases, maintenance of conditions between calibration and characterization experiments may be difficult to achieve in practice.

(ii) Measurements of size and quantities derived from it may be corrupted, if multilamellarity is not accounted for on the basis of individual vesicles (or a priori absent in the sample). Typically, the fraction of multilamellar vesicles in a sample is determined independently, if at all, using cryo-TEM, which does not allow identification of such vesicles at the level of individuals in the fluorescence characterization. ${ }^{15,16,23}$

(iii) Instead, some studies have addressed the multilamellarity issue by using additional reagents. While quenchers may be used to reveal multilamellar vesicles, they render unilamellar vesicles invisible for downstream applications. ${ }^{14}$ Alternatively, by functionalizing the lipids in the vesicles and letting them bind spectrally different fluorophores after preparation, multilamellarity may be assessed. ${ }^{17}$

So far, however, no single method overcomes all these complications. We develop such a method here. This provides workers at the interface between nanotechnology and biology with a simple and robust tool for characterization of vesicles, which accounts for vesicle individuality in terms of size and structural properties and simultaneously overcomes the multiple shortcomings of established approaches. To this end, we use dual-color fluorescence labeling of vesicles - of surfaces (lamellae, bilayers) and lumens, respectively (Figure 1a) and a simple but rigorous analysis of microscopy images. Our protocol determines size, shape, lamellarity, and encapsulation efficiency of individual vesicles. It needs no separate calibration measurements, and it is not limited to a specific type of fluorescence microscopy.

Specifically, we found that (i) vesicles of different structural states (Figure 1a) coexist in samples; (ii) an identifiable fraction of vesicles in samples are ideal (Figure 1a) in the sense that they are unilamellar, spheres, and encapsulate a number of cargo molecules corresponding to their volume; (iii) we can use these ideal vesicles as benchmarks to calibrate recordings; (iv) doing that, we determine the size of ideal vesicles with precision; (v) for each non-ideal vesicle (Figure 1a), data determined from its image may allow classification of its 
structural state; (vi) the calibration involved amounts to fitting a straight line to data; and (vii) our results are consistent with cryo-TEM measurements.

Thus, calibration is done simultaneously with the characterization of vesicles, as an integral part of it. This ensures both ease of use and robustness, since no separate calibration experiment is needed, and no maintenance of experimental conditions is required between calibration and characterization.

Application of this tool should facilitate (a) that only well-characterized vesicles are used in data analyses, and (b) optimization of vesicle preparation for use in biotechnology and medicine, in particular for drug delivery.

In order to resolve the structural states of individual nano-size vesicles in an extruded sample (Figure 1a), ${ }^{24}$ we imaged them with laser-scanning confocal microscopy (Figure 1b). Isolated individual vesicles imaged as diffraction-limited spots, which we resolved spatially to analyze their distributions of intensity (Figure $\mathbf{1 b} \mathbf{b} \mathbf{h}$ and Figure S1). We demonstrated Gaussian distributions of intensity (Figure $\mathbf{1} \mathbf{c}-\mathbf{h}$ and Figures S1-S4), ${ }^{25}$ and thus determined reliably the intensity, $I$, captured with the Gaussian fit, and the spatial variance, $\sigma^{2}$, of the fitted Gaussian for both spots in each pair (Supporting Information, Figure S5). ${ }^{26,27}$ These four data, $\left(I_{\text {surface }}, I_{\text {lumen }}, \sigma_{\text {surface }}^{2}, \sigma_{\text {lumen }}^{2}\right)$, exhaust the information in a pair of spots. Using all four data simultaneously (i) calibrates measurements, and (ii) yields the structure of each vesicle in a sample, but (iii) differs from previously reported dual-color methods, which only used intensities. ${ }^{15,17,23}$

In order to discriminate between ideal and non-ideal vesicles, the two intensities suffice: For an ideal vesicle, $I_{\text {surface }}$ is proportional to $S_{\text {ves }}$, the surface area of the vesicle, and $I_{\text {lumen }}$ is proportional to $V_{\text {ves }}$, the volume of the vesicle,

$$
\begin{gathered}
I_{\text {surface }}=\frac{\alpha_{\text {surface }}}{4 \pi} S_{\text {ves }} \\
I_{\text {lumen }}=\frac{\alpha_{\text {lumen }}}{4 \pi / 3} V_{\text {ves }} .
\end{gathered}
$$


a

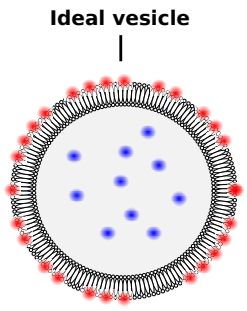

\section{Spherical}

Single lipid bilayer Optimal encapsulation.
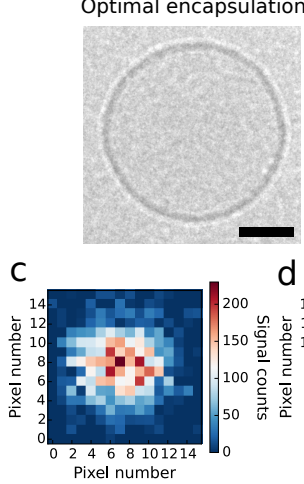

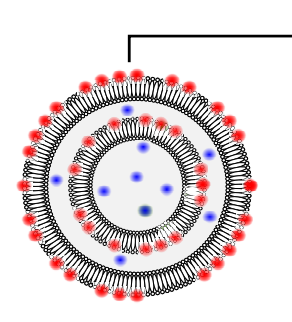

Non-ideal vesicles
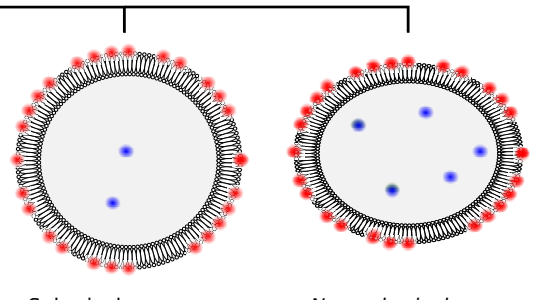

Single lipid bilayer

Single lipid bilayer Single lipid bilayer

Suboptimal encapsulation. Optimal encapsulation.

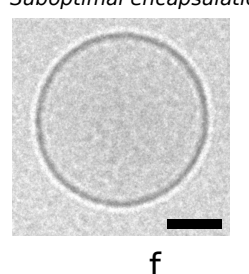

d

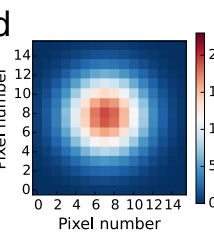

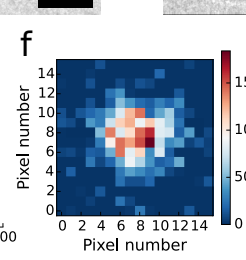

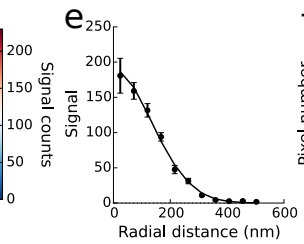

b

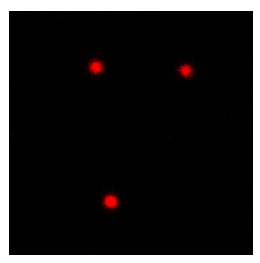

Surface channel

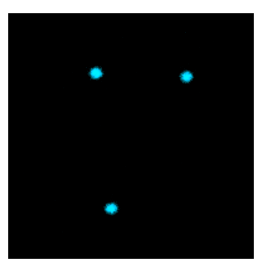

Lumen channel

9

$\mathrm{h}_{160}$
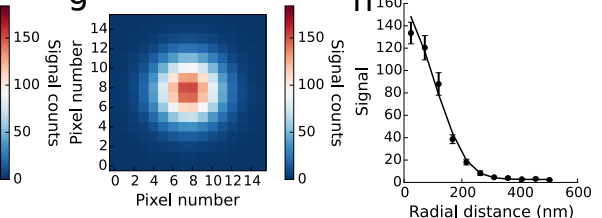

Figure 1. Structural properties of individual lipid vesicles that dual-color fluorescence images can reveal. (a) Lipid vesicles with bilayers fluorescently labeled with DiD fluorophores (red) and lumens enclosing Cfluoresceine water-soluble fluorophores (blue). An ideal vesicle is spherical, has a single lipid bilayer, and encapsulates the expected number of cargo molecules for a sphere its size. A non-ideal vesicle is non-spherical and/or has multiple bilayers and/or encapsulates a lower-than-expected number of cargo molecules. Cryo-TEM images of individual vesicles are shown for comparison (Supporting Information). Note that cryo-TEM cannot reveal vesicles with suboptimal encapsulation and/or deviations from spherical shape along the optical axis. Scale bar is $50 \mathrm{~nm}$. (b) Dual-color confocal fluorescence images of individual vesicles. The difference in excitation wavelength between the lumen and surface labels was exploited to image them sequentially. (c-h) The two intensity distributions from a doubly-labeled single vesicle. (c) Confocal microscopy image of an isolated lipid vesicle with DiD fluorophores labeling its lipid bilayer. The pixel size was $48 \mathrm{~nm}$. (d) Expected pixel output signals of a 2D Gaussian intensity distribution with parameters obtained by least-squares fitting to the image in c (Supporting Information). This determined the spot's total intensity and its spatial variance. (e) Comparison of radial distributions. Centered on the result of the localization analysis in $\mathbf{d}$, we constructed concentric annuli. For each annulus, we calculated the mean and s.e.m. of output signals from pixels with centers within an annulus (black dots with error bars). These mean values agree with the radial distribution of the expected values (black curve). (f-h) Same as $\mathbf{c}-\mathbf{e}$ for imaging of the Cfluoresceine fluorophores in the vesicle's lumen. Figure S1 gives more details. 
In both these identities, $\sigma_{\mathrm{PSF}}^{2}$ depends on the wavelength of the light, the numerical aperture of the objective, and on the focal depth at which the image is recorded but is the same for all vesicles in a single recording. ${ }^{25,28-32}$ It helps that confocal microscopy is less sensitive to focus ${ }^{28,29}$ than other microscopies. ${ }^{30-32}$ The variances $\sigma_{\text {dye }}^{2}$ depend on vesicle size and shape; for ideal vesicles $\sigma_{\text {dye,surface }}^{2}=R_{\text {ves }}^{2} / 3$ and $\sigma_{\text {dye,lumen }}^{2}=R_{\text {ves }}^{2} / 5$ (Supporting Information). Consequently,

- in a plot of $\sigma_{\text {surface }}^{2}$ against $I_{\text {surface }}$, ideal vesicles fall on a straight line with slope 
$\alpha_{\text {surface }}^{-1} / 3$ and second-axis intercept $\sigma_{\mathrm{PSF} \text {,surface }}^{2}$ (blue arrows, Figure $\mathbf{2 b}$ ). Thus, a fit of a straight line to these data calibrates the surface-channel by returning values for $\alpha_{\text {surface }}$ and $\sigma_{\mathrm{PSF}, \text { surface }}^{2}$ (Supporting Information).

- in a plot of $\sigma_{\text {lumen }}^{2}$ against $I_{\text {lumen }}^{2 / 3}$, ideal vesicles fall on a straight line with slope $\alpha_{\text {lumen }}^{-2 / 3} / 5$ and second-axis intercept $\sigma_{\mathrm{PSF}, \text { lumen }}^{2}$ (blue arrows, Figure 2c). Thus, a fit of a straight line to these data calibrates the lumen-channel by returning values for $\alpha_{\text {lumen }}$ and $\sigma_{\mathrm{PSF}, \text { lumen }}^{2}$ (Supporting Information).

- in a plot of $\sigma_{\text {lumen }}^{2}$ against $\sigma_{\text {surface }}^{2}$, ideal vesicles fall on a straight line with slope $3 / 5$ starting in $\left(\sigma_{\mathrm{PSF}, \text { surface }}^{2}, \sigma_{\mathrm{PSF}, \text { lumen }}^{2}\right)$ (blue arrows, Figure 2d). Falling on that straight line is a criterion for being ideal which is independent of that in Figure 2a.
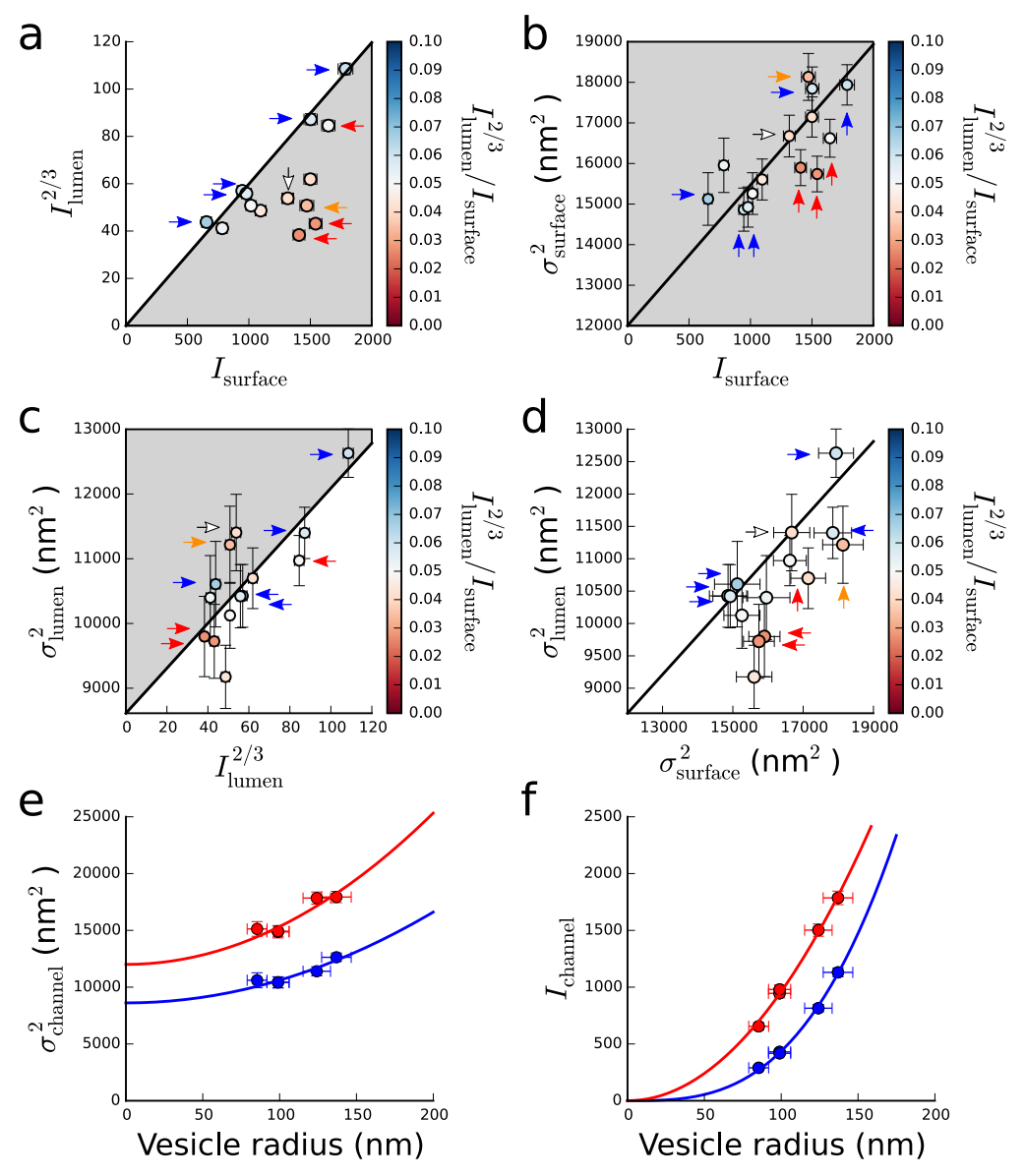

Figure 2. (Caption on the next page.) 
Figure 2. Raw data from a single dual-color recording characterize every recorded vesicle. Error bars were calculated by assuming that finite photon statistics was the only source of variation (Figure S1, Supporting Information). (a-d) Various displays of the raw data, $\left(I_{\text {surface }}, I_{\text {lumen }}^{2 / 3}, \sigma_{\text {surface }}^{2}, \sigma_{\text {lumen }}^{2}\right)$, obtained for each vesicle in a sample (Supporting Information). In each panel, the black, straight diagonal line is the projection to the panel of the straight line in four dimensions, equation (5), that was fitted to all four data values for all five ideal vesicles (blue arrows). Apart from stochastic errors, those five vesicles fall on top of the black line in each panel, which confirms their ideal nature. In quantitative terms, the straight line fitted in $4 \mathrm{D}$ to data for ideal vesicles fits with $p=0.47$ in a $\chi^{2}$ goodness-of-fit test. Some other vesicles fall away from the black lines, in directions that reveal the nature of their non-ideality. The value of the ratio $A=I_{\text {lumen }}^{2 / 3} / I_{\text {surface }}$ is indicated for each vesicle (color bar). Ideal vesicles maximize this ratio (blue shades). (a) Non-ideal vesicles have smaller values for $A$ and hence fall under the black line (grey area). (b) Suboptimal encapsulation efficiency does not affect the surface channel parameters, presumably, why such vesicles should fall near the black line. Non-ideal vesicles do not fall in a specific region, as multi-lamellar vesicles have higher intensity for given variance than ideal vesicles, while non-spherical vesicles have larger variance for given intensity. (c) Spherical multilamellar vesicles fall on the black line, if multi-lamellarity does not affect $I_{\text {lumen }}$ and $\sigma_{\text {lumen }}^{2}$, as presumed. Other non-ideal vesicles fall in the grey region (up to stochastic errors), as non-spherical vesicles have larger variance for given intensity, while suboptimal encapsulation results in lower intensity for given variance. Comparison of $\mathbf{a}-\mathbf{c}$ identifies vesicles that are multi-lamellar (red arrows), suboptimal encapsulators (white arrow), or non-spherical (orange arrow). (e) Measured variances versus radii determined by fit of equation (5) for the five ideal vesicles. Vesicle radii (circles) scatter around the projection of the fit (lines) for lumen (blue) as well as surface (red) channels. Thus individual vesicle radii were determined with $7 \%$ error. (f) Same as e for the measured intensities versus vesicle radii.

With both channels calibrated using data for the same ideal vesicles, each channel will provide a value for the radius of an ideal vesicle. Rather than combining these two independent values into a single estimate for each vesicle, we used the statistically optimal procedure, which fits the theory to all the data simultaneously, thus making use of all a priori information, including the fact that both color channels look at the same vesicles (Figure 1b).

This optimal fit amounts to weighted least-squares fitting of a straight line in four dimensions. Parameterized by $R_{\mathrm{ves}}^{2}$, the line 


$$
\left[\begin{array}{c}
\left\langle I_{\text {surface }}\right\rangle \\
\left\langle I_{\text {lumen }}^{2 / 3}\right\rangle \\
\left\langle\sigma_{\text {surface }}^{2}\right\rangle \\
\left\langle\sigma_{\text {lumen }}^{2}\right\rangle
\end{array}\right]=\left[\begin{array}{c}
0 \\
0 \\
\sigma_{\mathrm{PSF}, \text { surface }}^{2} \\
\sigma_{\mathrm{PSF}, \text { lumen }}^{2}
\end{array}\right]+\left[\begin{array}{c}
\alpha_{\text {surface }} \\
\alpha_{\text {lumen }}^{2 / 3} \\
1 / 3 \\
1 / 5
\end{array}\right] R_{\mathrm{ves}}^{2}
$$

is fitted to our four-dimensional single-vesicle data, $\left(I_{\text {surface }}, I_{\text {lumen }}^{2 / 3}, \sigma_{\text {surface }}^{2}, \sigma_{\text {lumen }}^{2}\right)$ (Supporting Information). This fit to data from the ideal vesicles calibrates the experiment by returning values for $\left(\alpha_{\text {surface }}, \alpha_{\text {lumen }}, \sigma_{\mathrm{PSF} \text {,surface }}^{2}, \sigma_{\mathrm{PSF}, \text { lumen }}^{2}\right)$. The fitted line delineates the expected values (denoted by $\langle\cdot\rangle$ ) for our data, and the differences between the fitted line and the data is due to finite photon number statistics, we assume. (This assumption is confirmed by the quality of the resulting fit: It has $p=0.47$ in a $\chi^{2}$ goodness-of-fit test.) The squared radius of a given vesicle is estimated by the value of $R_{\mathrm{ves}}^{2}$ corresponding to the point on this straight line that is closest to the data point of the vesicle (Supporting Information). The four coordinates of that point are the expected values for that vesicle's data.

Figure $\mathbf{2} \mathbf{a}-\mathbf{d}$ shows projections of the resulting fit onto two-dimensional subspaces as straight lines. Notice how data points corresponding to ideal vesicles scatter near the fitted line in each projection (blue arrows, Figure $\mathbf{2 a}-\mathbf{d}$ ). Thus, vesicles selected as ideal on the basis of intensities (Figure 2a) are consistently ideal also in other dimensions (Figure 2 bd).

In the recording considered in Figure 2, we found vesicle radii in the range from $85 \mathrm{~nm}$ to 137 nm (Figure 2e,f). Furthermore, data confirm the expected relationships between vesicle radii and vesicle data (Figure 2e,f). Note that the radii of individual ideal vesicles were determined with only $7 \%$ error from a single recording of their dual-colored spots (Figure 2).

We repeated this analysis for two other recordings that each contained at least four ideal vesicles (Supporting Information). This repetition demonstrated that the four calibration parameters, $\left(\alpha_{\text {surface }}, \alpha_{\text {lumen }}, \sigma_{\mathrm{PSF}, \text { surface }}^{2}, \sigma_{\mathrm{PSF}, \text { lumen }}^{2}\right)$ vary between recordings $($ Figure $\mathbf{3 a}-\mathbf{d})$, 
presumably due to different focal depths. Radii of individual, ideal vesicles from all three recordings (Figure 3e) were determined with high precision from the analysis of the individual recording. The range of measured radii is consistent with the distribution of radii obtained with cryo-TEM (Figure 3f, Supporting Information). Note, though, that the smaller vesicles did not yield sufficient fluorescence signals to permit analysis (Supporting Information).

We cannot directly compare data values $\left(I_{\text {surface }}, I_{\text {lumen }}, \sigma_{\text {surface }}^{2}, \sigma_{\text {lumen }}^{2}\right)$ from different recordings because these data were obtained under different conditions, as revealed by the different calibrations they result in (Figure $\mathbf{3 a}-\mathbf{d})$. We can remove this calibration-dependence by transforming each vesicle's data as done on the left-hand-side here,

$$
\left[\begin{array}{c}
I_{\text {surface }} / \alpha_{\text {surface }} \\
I_{\text {lumen }}^{2 / 3} / \alpha_{\text {lumen }}^{2 / 3} \\
3\left(\sigma_{\text {surface }}^{2}-\sigma_{\mathrm{PSF}, \text { surface }}^{2}\right) \\
5\left(\sigma_{\text {lumen }}^{2}-\sigma_{\mathrm{PSF}, \text { lumen }}^{2}\right)
\end{array}\right]=\left[\begin{array}{c}
S_{\text {ves }} /(4 \pi) \\
\left(V_{\text {ves }} /(4 \pi / 3)\right)^{2 / 3} \\
3 \sigma_{\text {dye,surface }}^{2} \\
5 \sigma_{\text {dye,lumen }}^{2}
\end{array}\right] .
$$

As the right-hand side shows, the result is four physical characteristics of the vesicle, and hence directly comparable for vesicles from different recordings or experiments (Figure 4).

For ideal vesicles, the four elements on the right-hand side all equal $R_{\text {ves }}^{2}$ up to finite photon statistics. Consequently, ideal vesicles from all three recordings fall on the same diagonal line in Figure $\mathbf{4 a -} \mathbf{d}$, and radii of ideal vesicles fall on the same curves in Figure $4 \mathbf{e}, \mathbf{f}$ when plotted against transformed variance and intensity data, respectively.

The four values in equation (6) are independent data except for their common calibration. Significant differences between their four values flag a non-ideal vesicle and suggest the nature of its non-ideality. Thus,

- values of $A$ (or $A$ transformed, $\alpha_{\text {surface }} / \alpha_{\text {lumen }}^{2 / 3} A$ ) discriminate between ideal and nonideal vesicles (Figure 2a and 4a). 

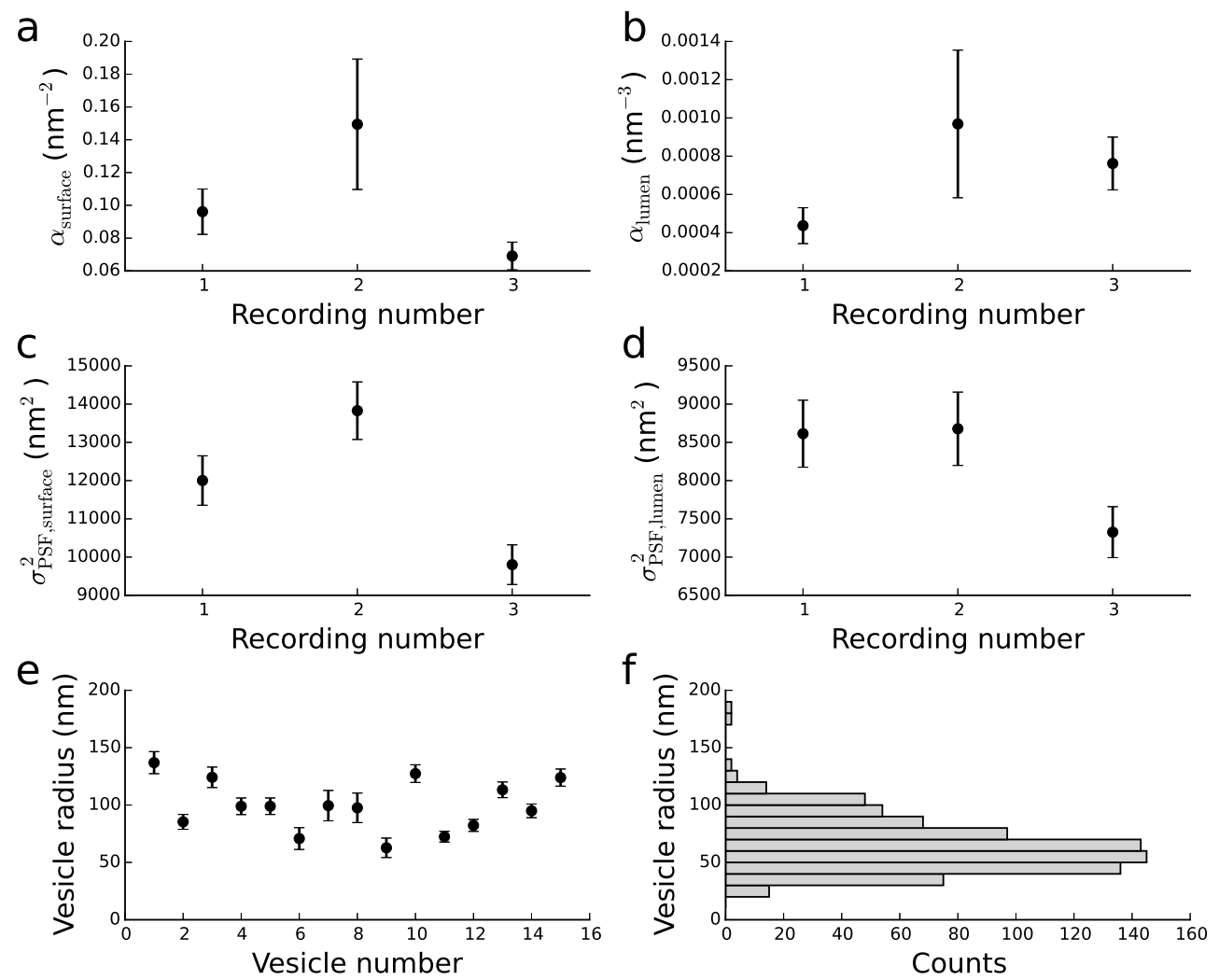

Figure 3. Radii of individual vesicles are determined reliably from individual recordings. (a-d) Parameter values from each of three time-lapse recordings of samples containing, respectively, five (Figure 2), four, and six ideal vesicles. Differences between parameter values from different recordings are statistically significant and presumably due to different focal depths, which potentially affects both the variance of the PSF and the excitation intensity. Error bars were calculated by assuming that finite photon statistics was the only source of variation (Supporting Information). (e) Radii of the vesicles determined by calibrating individual recordings (Supporting Information). (f) Distribution of radii for spherical vesicles observed using cryo-TEM (Supporting Information). Notice that the values determined in e overlap with this distribution, as expected. We note that the smallest vesicles did not yield sufficient signal to permit fluorescence analysis (Supporting Information). 
- in the surface-channel (Figures $\mathbf{2 b}$ and $\mathbf{4 b}$ ), compared to ideal vesicles, (i) multilamellar vesicles have larger intensity for given variance; (ii) non-spherical vesicles have larger variance for given intensity; while (iii) suboptimal encapsulation efficiency has no effect.

- In the lumen-channel, non-ideal vesicles fall in the grey region in Figures 2c and 4c because (i) suboptimal encapsulation efficiency yields a lower intensity for a given volume and corresponding variance; (ii) non-spherical shape yields a larger variance for given intensity; and (iii) multi-lamellarity has no effect.

Non-ideal vesicles identified in Figure 2a we classified by how they plot away from the diagonal black lines in Figure 2b,c. Since a vesicle's intensities are determined with errors that are negligible compared to errors on its variances (Figure $2 \mathbf{b}, \mathbf{c}$ ), it is the distance along the variance-axis between its plotting symbol and the black line in Figure $\mathbf{2 b , c}$ that requires interpretation. Is it due to experimental noise or to non-ideal vesicle properties? In both channels we considered this distance in units of its theoretical error for an ideal vesicle. If it exceeded 1.5 in a channel, the vesicle was flagged as non-ideal in that channel. For Gaussian errors and independent channels, 14 per cent of such flags are false (Type I errors) if the null hypothesis is true, i.e., if the vesicle appears ideal in the channel considered.

We used this to identify spherical vesicles that are multi-lamellar (Figure $\mathbf{2 a}-\mathbf{d}$, red arrows), spherical vesicles with suboptimal encapsulation efficiency (Figure $\mathbf{2 a}-\mathbf{d}$, white arrow), and vesicles with non-spherical shapes (Figure $\mathbf{2} \mathbf{a}-\mathbf{d}$, orange arrow).

Among these vesicles, we can determine the size of the multi-lamellar ones and the suboptimal encapsulators, because they are spherical and appear ideal in one channel. Their sizes are determined from their data in the channel in which they appear ideal, using that channel's calibration based on ideal vesicles. The lumen-channel provides the size of spherical, multi-lamellar vesicles, and the surface channel provides the size of spherical, suboptimal encapsulators (Supporting Information). Thus, we found radii of $82 \mathrm{~nm}, 86 \mathrm{~nm}$, and $120 \mathrm{~nm}$ for the multi-lamellar vesicles and $117 \mathrm{~nm}$ for the suboptimal encapsulator (Fig- 

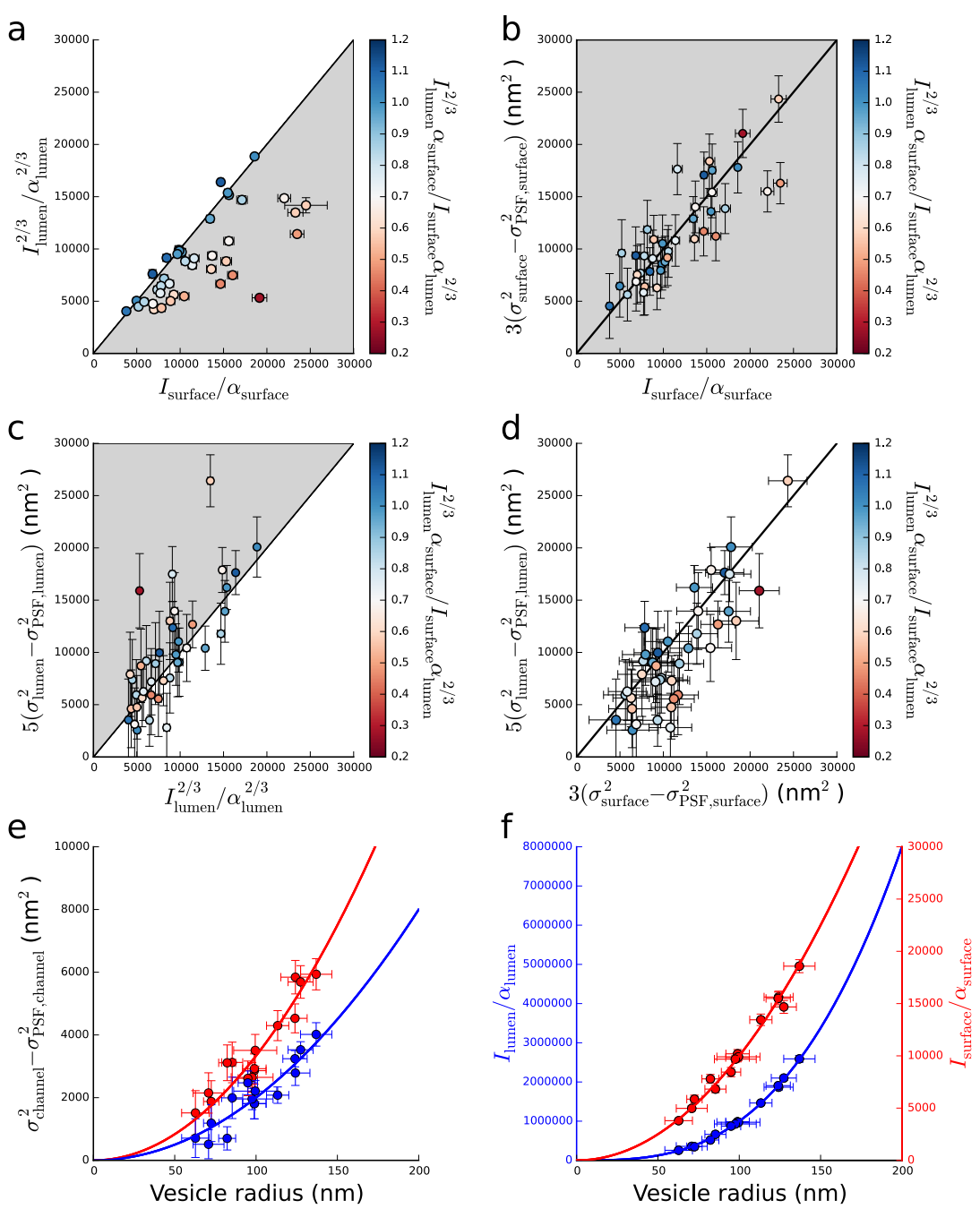

Figure 4. Rescaled vesicle data obtained from three recordings. (a-d) Vesicle data from three time-lapse recordings, after transformation as in equation (6), which removes dependence on calibration. The transformed intensity ratio, $\alpha_{\text {surface }} I_{\text {lumen }}^{2 / 3} /\left(\alpha_{\text {lumen }}^{2 / 3} I_{\text {surface }}\right)$, of each vesicle is indicated (color bar). In the three recordings, we identified a total of 15 ideal vesicles (cold colors). They fall on (or near) the straight line with unit slope passing through the origin in each panel (black line). Non-ideal vesicles fall in the grey regions, as predicted by theory (see Figure 2). (e) Radii of the 15 ideal vesicles, determined by calibrating three individual recordings. Measured variances versus vesicle radii (circles) scatter around their theoretical relationship, equation (6), in both channels: lumen (blue) and surface (red). (f) Same as e for the measured intensities versus vesicle radii.

ure $\mathbf{2} \mathbf{a}-\mathbf{d}$, red and white arrows). If instead one ignores multi-lamellarity and proceeds blindly with the measured surface intensities in conjunction with equation (1), then one overestimates the radii of these multilamellar vesicles by, respectively, $48 \%, 9 \%$, and $47 \%$. 


\section{Thus, ignoring multi-lamellarity may lead to substantial errors on individual vesicle sizes and} quantities derived from them. In general, multi-lamellarity comes in various forms, ranging from vesicles enclosing other small vesicles to vesicles with multiple concentric lipid bilayers (Figure S6). The error associated with ignoring multi-lamellarity depends on the area of the extra lamella(e). The error is approximately $40 \%$ for the case illustrated in Figure 1a of a vesicles with two concentric bilayers of almost identical radii.

Vesicles that are non-ideal in both channels in ways not explained as non-spherical we cannot classify. Vesicles that are not non-ideal in both channels are nevertheless non-ideal if Figure 2a says so. They just appear ideal in both channels due to the large error bars on their variances.

Cryo-TEM demonstrated that our vesicle sample is, indeed, heterogeneous (Figures 1a, 3f, and S6, Supporting Information), in agreement with our results in Figures 24. Specifically, with cryo-TEM we observed that $\sim 50 \%$ of vesicles in our type of sample consisted of more than one bilayer or contained additional smaller vesicles, $\sim 20 \%$ of vesicles appeared non-spherical, and just $\sim 40 \%$ of vesicles were both unilamellar and appeared spherical. This agrees strikingly well with the $\sim 40 \%$ of vesicles we found to be ideal in our fluorescence measurements (Figure 4). Both these numbers represent upper limits for the fraction of ideal vesicles in our sample, however: Cryo-TEM is incapable of detecting suboptimal encapsulators and vesicles with shapes that differ from spheres along the optical axis only, while our protocol can do that. On the other hand, our protocol is incapable of detecting both small and large effects of multi-lamellarity (Supporting Information) and/or small deviations from a spherical shape, due to limited resolution.

In summary, we have demonstrated how structural properties and sizes of individual vesicles may be obtained using simple dual-color fluorescence microscopy in conjunction with simple but rigorous image data analysis. Applying this to extruded samples, we observed structural variation among vesicles and thus found that only a fraction of vesicles are ideal. Vesicle ideality is commonly assumed for every vesicle in a sample, why such structural 
variation may affect interpretations of results from established and new analyses that do not take it into account.

Importantly, our identification of ideal vesicles based on two-color intensities is insensitive to any perturbation in vesicle properties that do not change the number of fluorophores in the vesicle's surface/lumen after its preparation. Such effects include, but are not limited to, changes of vesicle shape/volume, e.g., induced by attachment strategy or proximity to a surface. In the present study, such effects were negligible, since the vesicles classified as ideal based on intensities appeared ideal also in the other dimensions spanned by the four data determined for each vesicle. This highlights another use of our protocol's built-in diagnostics (Figure 2a-d): it warns the user about variations in data not explained by the sample heterogeneities considered here. For example, if the fraction of lipids labelled had varied between vesicles in the sample, ${ }^{17}$ ideal vesicles would not fall on the line with maximal slope that relates the two intensities of fluorescence (Figures $\mathbf{2 a}$ and $\mathbf{4 a}$ ).

Thus, the surface immobilization used here provides an optimal platform for benchmarking of our protocol, and surface immobilization is a critical element in many experiments. ${ }^{2}$ Surface immobilization is not critical to our protocol, however. Our protocol is so simple that it should work also for unattached vesicles if they are confined in their vertical motion and move negligibly laterally within the shutter time. This could be useful in contexts where vesicles are free to move, either due to the specific nature of an assay or because they cannot be functionalized, which prevents specific attachment to a surface and hence prevents the use of our protocol in the form presented here. Micro/nano-fluidic devices could provide a suitable platform for such studies, we expect. ${ }^{22}$ In such devices our protocol would complement other ways to infer sizes of vesicles, such as diffusion-based approaches.

The simplicity of our protocol makes it easy to implement in any pipeline of analysis of vesicles with radii below $150 \mathrm{~nm}$. Its independence of external calibration contributes to this ease, ensures robustness, and eliminates potential errors, since its calibration parameters are determined from the same single-vesicle data as are used to characterize the vesicles, with a 
new calibration for each new recording in the characterization study. External calibration of the four calibration parameters can, however, improve the resolution of the method beyond the resolution demonstrated here. One might, e.g., mix the sample of vesicles with small, well-characterized, brightly fluorescent objects to provide an independent calibration of the PSF variance at the focal depth of each recording.

It would be interesting to extend our protocol to vesicles that are non-spherical by design and/or carry solid cargo. An extension to non-spherical vesicles with simple, mathematically defined shapes should be relatively straightforward. The orientation of non-spherical vesicles relatively to the image plane matters and should be known, ideally. For ellipsoids, prolate or oblate, attractive interactions with a surface parallel to the image plane might ensure that the major axis/axes are approximately parallel to the image plane. Pegylated vesicles loaded with doxorubicin sulfate crystals provide an example of practical interest. (One version of this carrier system currently is marketed as Doxil.) These nano-size vesicles are elongated by their crystalline cargo, which makes them approximately prolate ellipsoids. ${ }^{12,33}$ The presence of the solid cargo complicates the analysis, however. Doxorubicin is naturally fluorescent, but its fluorescence is sensitive to surroundings and shielded by the lipid bilayer. ${ }^{34}$ If its fluorescence nevertheless can be harnessed as a reliable signal in a third color channel, an error analysis based on feasible strengths of fluorescence signals can reveal a priori which questions about the system might be answered experimentally, because, as demonstrated here for spherical vesicles, photon shot-noise alone explains the experimental errors in our protocol.

In conclusion, the versatile nature of our protocol should make it widely applicable. Its ability to identify ideal vesicles, in particular, could optimize assays and production of vesicles. 


\section{Author Information}

K.I.M. and N.E. conceived the idea. K.I.M. and H.F. designed research and developed methods of analysis. K.I.M. analyzed the data. H.F. supervised the theoretical analysis. C.T. performed the experiments. N.E. and T.L.A. supervised the experimental work. K.I.M., T.L.A., and H.F. wrote the manuscript. All authors read and approved the final manuscript.

\section{Notes}

The authors declare no competing financial interests.

\section{Acknowledgement}

This work was supported by a Lundbeck Foundation Center of Excellence (R155-2013-14113) (to T.L.A.) and the Novo Nordisk Foundation Challenge Programme (NNF16OC0022166) (to T.L.A. and H.F.) We thank J.B. Larsen for discussions, M.F. de Val Weywadt and P. Kempen for assisting us with the acquisition of the cryo-TEM measurements, and R. Marie and M.F. Hansen for reading the manuscript.

\section{Supporting Information Available}

The following files are available free of charge.

- SupportingInformation.pdf: Experimental and theoretical methods, theory, error analysis, and supporting figures.

- SupportingSoftware.zip: Software including test data.

\section{References}

(1) Kirchhausen, T. Nat. Cell Biol. 2012, 14, 906-908. 
(2) Veshaguri, S.; Christensen, S. M.; Kemmer, G. C.; Ghale, G.; Møller, M. P.; Lohr, C.; Christensen, A. L.; Justesen, B. H.; Jørgensen, I. L.; Schiller, J.; Hatzakis, N. S.; Grabe, M.; Pomorski, T. G.; Stamou, D. Science 2016, 351, 1469-1473.

(3) Larsen, J. B.; Jensen, M. B.; Bhatia, V. K.; Pedersen, S. L.; Bjørnholm, T.; Iversen, L.; Uline, M.; Szleifer, I.; Jensen, K. J.; Hatzakis, N. S.; Stamou, D. Nat. Chem. Biol. 2015, 11, 192-194.

(4) Chiu, D. T.; Wilson, C. F.; Ryttsén, F.; Strömberg, A.; Farre, C.; Karlsson, A.; Nordholm, S.; Gaggar, A.; Modi, B. P.; Moscho, A.; Garza-López, R. A.; Orwar, O.; Zare, R. N. Science 1999, 283, 1892-1895.

(5) Okumus, B.; Wilson, T. J.; Lilley, D. M. J.; Ha, T. Biophys. J. 2004, 87, 2798-806.

(6) Sato, Y. T.; Umezaki, K.; Sawada, S.; Mukai, S.-a.; Sasaki, Y.; Harada, N.; Shiku, H.; Akiyoshi, K. Sci. Rep. 2016, 6, 21933.

(7) Gibbs, B. F.; Kermasha, S.; Alli, I.; Mulligan, C. N.; Gibbs, F.; Kermasha, S.; Inteaz Al, B. Int. J. Food Sci. Nutr. 1999, 50, 213-24.

(8) Bourzac, K. Nature 2012, 491, 558-560.

(9) Andresen, T. L.; Jensen, S. S.; Jørgensen, K. Prog. Lipid Res. 2005, 44, 68-97.

(10) Roberts, D. L.; Ma, Y.; Bowles, S. E.; Janczak, C. M.; Pyun, J.; Saavedra, S. S.; Aspinwall, C. A. Langmuir 2009, 25, 1908-1910.

(11) Moghimi, S. M.; Hunter, A. C.; Andresen, T. L. Annu. Rev. Pharmacol. Toxicol. 2012, 52, 481-503.

(12) Yingchoncharoen, P.; Kalinowski, D. S.; Richardson, D. R. Pharmacol. Rev. 2016, 68, $701-787$.

(13) Chen, C.; Zhu, S.; Huang, T.; Wang, S.; Yan, X. Anal. Methods 2013, 5, 2150-2157. 
(14) Heider, E. C.; Barhoum, M.; Edwards, K.; Gericke, K.-H.; Harris, J. M. Anal. Chem. 2011, 83, 4909-4915.

(15) Kunding, A. H.; Mortensen, M. W.; Christensen, S. M.; Stamou, D. Biophys. J. 2008, 95, 1176-1188.

(16) Lohr, C.; Kunding, A. H.; Bhatia, V. K.; Stamou, D. In Methods Enzymol. Liposomes; Duzgunes, N., Ed.; Elsevier, 2009; Vol. 465; pp 143-160.

(17) Larsen, J.; Hatzakis, N. S.; Stamou, D. J. Am. Chem. Soc. 2011, 133, 10685-10687.

(18) Mui, B.; Cullis, P.; Evans, E.; Madden, T. Biophys. J. 1993, 64, 443-453.

(19) Heider, E. C.; Peterson, E. M.; Barhoum, M.; Gericke, K.-H.; Harris, J. M. Anal. Chem. 2011, 83, 5128-5136.

(20) Edwards, K. A.; Baeumner, A. J. Talanta 2006, 68, 1432-1441.

(21) Olsson, T.; Zhdanov, V. P.; Höök, F. J. Appl. Phys. 2015, 118, 064702.

(22) Friedrich, R.; Block, S.; Alizadehheidari, M.; Heider, S.; Fritzsche, J.; Esbjörner, E. K.; Westerlund, F.; Bally, M. Lab Chip 2017, 17, 830-841.

(23) Lohse, B.; Bolinger, P. Y.; Stamou, D. J. Am. Chem. Soc. 2008, 130, 14372-14373.

(24) Olson, F.; Hunt, C. A.; Szoka, F. C.; Vail, W. J.; Papahadjopoulos, D. Biochim. Biophys. Acta, Biomembr. 1979, 55\%, 9-23.

(25) Zhang, B.; Zerubia, J.; Olivo-Marin, J.-C. Appl. Opt. 2007, 46, 1819-1829.

(26) Mortensen, K. I.; Churchman, L. S.; Spudich, J. A.; Flyvbjerg, H. Nat. Methods 2010, 7, 377-381.

(27) Mortensen, K. I.; Flyvbjerg, H. Sci. Rep. 2016, 6, 28680.

(28) Wilson, T. J. Microsc. 2011, 244, 113-121. 
(29) Cole, R. W.; Jinadasa, T.; Brown, C. M. Nat. Protoc. 2011, 6, 1929-1941.

(30) Mortensen, K. I.; Sung, J.; Flyvbjerg, H.; Spudich, J. A. Nat. Commun. 2015, 6, 8621.

(31) Mortensen, K. I.; Sung, J.; Spudich, J. A.; Flyvbjerg, H. In Methods Enzymol. Singlemolecule enzymology: Fluorescence-Based and High-Throughput Methods; Spies, M., Chemla, Y. R., Eds.; Elsevier, 2016; Vol. 581; pp 147-185.

(32) Proppert, S.; Wolter, S.; Holm, T.; Klein, T.; van de Linde, S.; Sauer, M. Opt. Express 2014, 22, 10304-10316.

(33) Andriyanov, A. V.; Koren, E.; Barenholz, Y.; Goldberg, S. N. PLoS One 2014, 9, e92555.

(34) Karukstis, K. K.; Thompson, E. H.; Whiles, J. A.; Rosenfeld, R. J. Biophys. Chem. 1998, 73, 249-263. 


\section{Graphical TOC Entry}

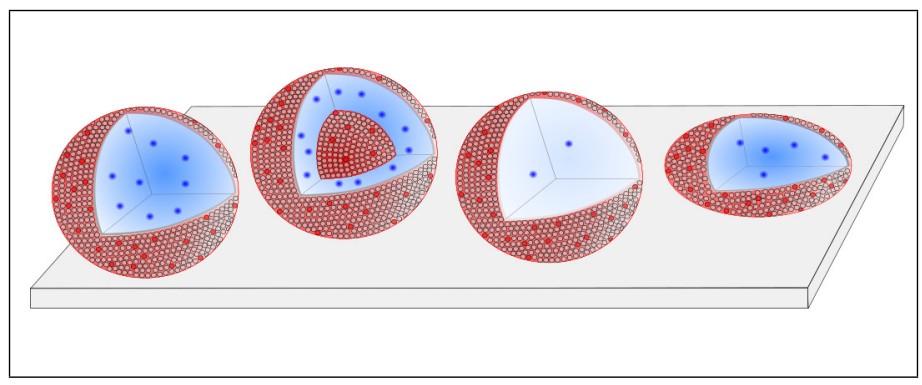

